\title{
Alpha-2 macroglobulin is the simplest serum biomarker for liver fibrosis and fibrogenesis in chronic hepatitis $\mathrm{C}$
}

\author{
Atanasova, Evelina ${ }^{1}$, Martinova, Fani $^{2}$, Jelev, Deyan ${ }^{1}$, \\ Antonov, Krassimir ${ }^{1}$, de Mey, Christian ${ }^{3}$, Mateva, \\ Ludmila ${ }^{1}$, Krastev, Zahary'.
}

1. Clinic of Gastroenterology, University Hospital "St. Ivan Rilski", Sofia, Bulgaria;

2. Department of transfusion hematology and immunology, University Hospital "Pirogov", Sofia, Bulgaria;

3. ACPC - Applied Clinical Pharmacology Services, Mainz-Kastel, Germany;

\section{Abstract}

Systemic inflammatory response triggered by HCV per se and/or its subsequent immune cascades and acute phase inflammatory proteins may play a major role in it. In inflammatory or injured liver, the increase of A2MG inhibits catabolism of matrix proteins and thus causes liver fibrosis. Objective: 129 patients with chronic HCV infection were studied. All of them were candidates for combined treatment with pegylated interferon-alpha. Methods: Alpha 2 macroglobulin and haptoglobin levels were measured by immuno-turbidimetry. Serum concentrations of pro-hepcidin, hsCRP and TNF-alpha were measured by commercially available ELISA kits. Serum HCV RNA was quantified by using real-time PCR assay. Liver biopsy was obtained applying Menghini's technique and was evaluated by METAVIR system. For non-invasive assessment of liver fibrosis and fibrogenesis we used specific scoring systems (APRI, FIB-4, SOS-FS and GAMAGEN). 
Results: The mean A2MG level was significantly lower in subjects with $A 1$ vs. $A 2(p<0,001)$ and A3 $(p<0,001)$. Patients with F3 stage of fibrosis were with higher mean A2MG level compared to those with F1 $(p<0.001)$ and F2 $(p<0.05)$. A2MG positively correlated with hepatic necroinflammation $(r=0.433 ; p=0.000)$ and fibrosis $(r=0.325 ; p=0.000)$. A moderate positive correlation between $\mathrm{A} 2 \mathrm{MG}$ and patient age is very important point as fibrosis rapidly progresses in elder patients.

Conclusion: A2MG is quite an informative serum biomarker that reflects liver fibrosis and fibrogenesis in chronic hepatitis $C$. It can be used in combination with haptoglobin, IgG and AST in scoring systems for non-invasive assessment of liver fibrosis and figrogenesis.

Keywords: hollow posts, pre-endodontic build-up, severely destructed teeth, provisionalisation

\section{Introduction}

Chronic hepatitis $\mathrm{C}(\mathrm{CHC})$ is associated with liver cell necrosis, inflammation, regeneration, fibrosis, and as a result of this approximately 20 to $30 \%$ of patients develop cirrhosis over a 20 to 30 year period of time $(1,2)$. Systemic inflammatory response triggered by HCV per se and/or its subsequent immune cascades and acute phase inflammatory proteins may play a major role in it $(3,4)$.

During the last 15 years it has been well established that hepatic necro-inflammatory activity and stages of liver fibrosis correspond to serum concentration of several biomarkers, e.g. $\alpha 2$ macroglobulin (A2MG), haptoglobin, gamma-globulin, pro-hepcidin, aspartate aminotransferase (AST) and platelet count (PIt) $(5,6,7,8)$.

In patients with cirrhosis, AST is higher than ALT (alanine aminotransferase) and platelets are low. Based on this fact a simple AST-to-platelet ratio index (APRI) was developed for non-invasive diagnosis of advanced fibrosis and cirrhosis (8). FIB-4 index includes also patient age in addition to aminotransferases and platelets, as liver fibrosis is more severe in elder patients $(9,10)$.

In the context of acute-phase proteins, A2MG is synthesized in hepatocytes and stellate cells (11-12). Both its hepatic and serum levels increase in fibrogenetic environment (13). In inflammatory or injured liver, the increase of A2MG inhibits catabolism of matrix proteins and thus causes liver fibrosis $(13,14)$. Haptoglobin is strongly and negatively associated with fibrosis $(5,15,16)$. Serum concentration of gamma-globulin is associated with cirrhosis and portosystemic shunts (17). A2MG, haptoglobin and globulin are well-known serum biomarkers of hepatic fibrosis and are significant components of FibroTest and Hepascore $(18,19)$. Developed by us SOS FS also includes A2MG and haptoglobin (20). In addition A2MG, IgG, AST and serum albumin were included in GAMAGEN, which is the only reported score for non-invasive assessment of liver fibrogenesis (20).

C-reactive protein (CRP) has been shown to be closely related to the occurrence of systemic inflammatory response (21). With respect to hepatological views, CRP is an acute-phase protein, and its expression in hepatocytes is closely related with pro-inflammatory cytokines, such as tumor necrosis factor-alpha (TNF$\alpha$ ), interleukin-1, and interleukin-6 $(3,22)$. There were discrepant results of the association of CRP level and anti-HCV seropositivity in different studies (23 -28). Tumor necrosis factor $\alpha$ (TNF- $\alpha)$ is a cytokine, produced primarily by activated monocytes and lymphocytes. Although low levels of TNF- $\alpha$ can contribute 
to cell protection, excessive amounts may cause cell damage. Raised serum TNF- $\alpha$ level has been shown in chronic hepatitis $C$ virus infection (29). Limited data are available regarding the association between A2MG and CRP, TNF-alpha or pro-hepcidin in CHC.

Histological activity of liver disease and stage of fibrosis are important predictors of disease progression and treatment outcome. Their precise verification is an obligate prerequisite before initiation of antiviral therapy (1). Liver biopsy is still considered the gold standard for the assessment of hepatic necroinflammatory activity and stage of liver fibrosis (1). However, after initial determination of liver necroinflammatory activity and fibrosis stage by biopsy, it is important to identify simple and widely available biomarkers that correlate well with liver morphology and are suitable for the monitoring of $\mathrm{CHC}$ patients in everyday clinical practice. Moreover, it is essential to identify patients with active fibrogenesis early, because they are at risk of rapid development of advanced fibrosis and fast progression to cirrhosis.

\section{Aim}

With this background we aimed to investigate the association between A2MG level and other acute phase proteins, liver morphology and some scoring systems for the non-invasive diagnosis of fibrosis.

\section{Materials and methods}

\section{Patients}

One hungered and twenty-nine patients with chronic HCV infection were studied. All of them were candidates for combined treatment with pegylated interferon-alpha (Peg-IFN and ribavirin - (Table 1)). 
Table 1. Characteristics of studied patients

\begin{tabular}{|c|c|c|}
\hline \multicolumn{3}{|c|}{ Patients 'number (n) 129} \\
\hline Age (years) & Mean \pm SD & $46.1 \pm 12.3$ \\
\hline Gender (n) & male/female & $56 / 73$ \\
\hline AST (U/L) & Mean \pm SD & $60.1 \pm 34.2$ \\
\hline $\mathrm{ALT}(\mathbf{U} / \mathrm{L})$ & Mean \pm SD & $88.9 \pm 50.9$ \\
\hline HCV RNA(IU/ml) & Mean \pm SD & $2601193 \pm 5810222$ \\
\hline Albumin (g/L) & Mean \pm SD & $47.0 \pm 3.6$ \\
\hline Platelet count (G/L) & Mean $\pm S D$ & $226.2 \pm 69.8$ \\
\hline Histological activity* (n) & $\begin{array}{l}\text { A0 } \\
\text { A1 } \\
\text { A2 } \\
\text { A3 } \\
\text { Unknown }\end{array}$ & $\begin{array}{l}0 \\
29 \\
68 \\
28 \\
4\end{array}$ \\
\hline 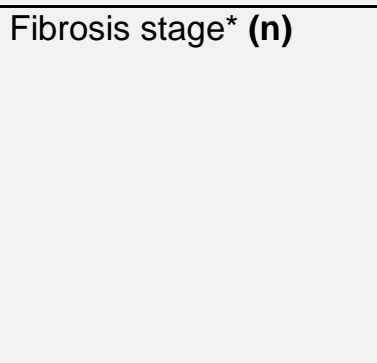 & $\begin{array}{l}\text { F0- } \\
\text { F1 } \\
\text { F2 } \\
\text { F3 } \\
\text { F4 } \\
\text { Unknown }\end{array}$ & $\begin{array}{l}0 \\
29 \\
49 \\
41 \\
6 \\
4\end{array}$ \\
\hline
\end{tabular}

\section{Measurements}

Serum HCV RNA was quantified by using real-time PCR assay (Roche Diagnostics). Routine lab methods were used for the testing of complete blood count, including platelets, as well as for serum levels of AST, ALT, albumin and lgG.

Alpha 2 macroglobulin, haptoglobin levels were measured by immuno-turbidimetry.

Serum concentrations of pro-hepcidin, hsCRP and TNF-alpha were measured by commercially available ELISA kits.

Only serum level of IgG, pro-hepcidin and TNF-alpha were not measured in the whole group of 129 patients: IgG was tested in 98 patients, while pro-hepcidin and TNF-alpha - in 31 subjects. 
Liver biopsy was obtained from 125 out of the 129 studied patients by applying Menghini's technique and was evaluated by the METAVIR system (30). Only 4 patients were without histological verification of liver disease activity and stage of fibrosis due to hemophilia A or coagulation abnormalities (Tabl. 1).

\section{Non-invasive indexes of liver fibrosis and fibrogenesis}

APRI was calculated by dividing the AST level $(U / L)$, expressed as the number of times above the upper limit of normal (ULN), by platelet count [G/L]: AST NLN x $100 /$ platelet count [G/L] (8).

FIB-4 was calculated using the formula: age [years] x AST [U/L]/(platelets $[G / L] \times A L T[U / L) 1 / 2)(10)$. SOS FS was calculated by using the formula: A2MG $[g / L]$ - haptoglobin $[g / L](20)$.

GAMAGEN was calculated by the formula: $\operatorname{lgG} / 14+\mathrm{AST} / 2 \times \mathrm{ULN}+\mathrm{A} 2 \mathrm{MG}[\mathrm{g} / \mathrm{l}] / 2.5$ - albumin $[\mathrm{g} / \mathrm{l}] / 41$ (20).

\section{Statistical Analysis}

Results are presented as the mean SD, counts, and percentages. All calculations were made using SPSS (version 11.0.4.0) software (SPPS Inc., Chicago, IL). A p value of less than 0.05 was considered statistically significant.

\section{Results}

Mean serum levels of measured acute phase proteins are presented in table 2.

Table 2. Mean serum levels of acute phase proteins

\begin{tabular}{|l|l|l|}
\hline Parameters & $\mathrm{N}$ & Mean \pm SD \\
\hline Alpha 2 macroglobulin (g/L) & $\mathrm{n}=129$ & $3.17 \pm 1.01$ \\
\hline Haptoglobin (g/L) & $\mathrm{n}=129$ & $0.94 \pm 0.5$ \\
\hline Pro-hepcidin (ng/mL) & $\mathrm{n}=31$ & $770.8 \pm 292.27$ \\
\hline IgG (g/L) & $\mathrm{n}=98$ & $13.58 \pm 2.24$ \\
\hline CRP (mg/L) & $\mathrm{n}=129$ & $1.67 \pm 2.46$ \\
\hline TNF-alpha (pg/mL) & $\mathrm{n}=31$ & $78.1 \pm 73.1$ \\
\hline
\end{tabular}

\section{$\underline{A 2 M G}$ and classical surrogate markers for the evaluation of chronic $\mathrm{CHC}$}

A positive correlation was found between A2MG levels and AST $(r=0.437 ; p=0.000)$, while correlations with ALT and platelet count were very low $(r<0.30)$, although they were significant. There was no correlation between A2MG and HCV RNA level (Table 3). 
Table 3. Correlation coefficients ( $r$ ) between A2MG and aminotransferases, HCV RNA and PLT

\begin{tabular}{|c|c|c|c|}
\hline Parameter & & A2MG & $\mathbf{P}$ \\
\hline AST & $(n=129)$ & 0.437 & 0.000 \\
\hline $\mathrm{ALT}$ & $(n=129)$ & 0.253 & 0.004 \\
\hline Plt & $(n=129)$ & -0.202 & 0.022 \\
\hline HCV RNA & $(n=129)$ & 0.023 & 0.792 \\
\hline
\end{tabular}

\section{A2MG and acute phase proteins}

There was a positive correlation between A2MG and IgG $(r=0.375 ; p=0.000)$. A2MG and haptoglobin correlated negatively $(r=-0.301 ; p=0.000)$. The correlation between A2MG and albumin, and globulin was very low. No correlation was found between A2MG and CRP, TNF-alpha and pro-hepcidin (Table 4).

Table 4. Correlation coefficients ( $r$ ) between A2MG and acute phase proteins

\begin{tabular}{|ll|l|l|}
\hline Parameters & & A2MG & P \\
\hline albumin & $(n=129)$ & -0.284 & 0.001 \\
\hline globulin & $(n=129)$ & 0.226 & 0.010 \\
\hline IgG & $(n=98)$ & $\mathbf{0 . 3 7 5}$ & $\mathbf{0 . 0 0 0}$ \\
\hline CRP & $(n=129)$ & -0.157 & 0.076 \\
\hline TNF-alpha & $(n=31)$ & 0.299 & 0.102 \\
\hline Haptoglobin & $(n=129)$ & $-\mathbf{0 . 3 0 1}$ & $\mathbf{0 . 0 0 0}$ \\
\hline Pro-hepsidin & $(n=31)$ & -114 & 0.539 \\
\hline
\end{tabular}

\section{$\underline{A 2 M G}$ and liver histology}

A2MG correlates significantly with both hepatic necro-inflammatory activity and stage of liver fibrosis (Table 5). 
Table 5. Correlation coefficients ( $r$ ) between A2MG and liver histology (METAVIR)

\begin{tabular}{|l|l|l|}
\hline Parameters & A2MG & P \\
\hline Histological activity $\quad(n=125)$ & $\mathbf{0 . 4 3 3}$ & $\mathbf{0 . 0 0 0}$ \\
\hline Stage of fibrosis & $\mathbf{0 . 3 2 5}$ & $\mathbf{0 . 0 0 0}$ \\
\hline
\end{tabular}

Mean A2MG levels among patients with A1; $\mathrm{A} 2$ and A3 grade of liver necro-inflammatory activity were $2.34 \pm 0,79 \mathrm{~g} / \mathrm{L} ; 3.36 \pm 0.90 \mathrm{~g} / \mathrm{L}$ and $3.64 \pm 1.01 \mathrm{~g} / \mathrm{L}$, respectively. The mean A2MG level was significantly lower in subjects with $A 1$ vs. $A 2(p<0,001)$ and $A 3(p<0,001)$ - figure $1 a$.

Mean A2MG levels in patients with F1; F2 and F3 fibrosis stage were $2.71 \pm 1.05 \mathrm{~g} / \mathrm{L} ; 3.14 \pm 0.94 \mathrm{~g} / \mathrm{L}$ and $3.53 \pm 0.96 \mathrm{~g} / \mathrm{L}$, respectively. Patients with $\mathrm{F} 3$ stage of fibrosis were with higher mean A2MG level compared to those with F1 $(p<0.001)$ and F2 $(p<0.05)$ - figure $1 b$

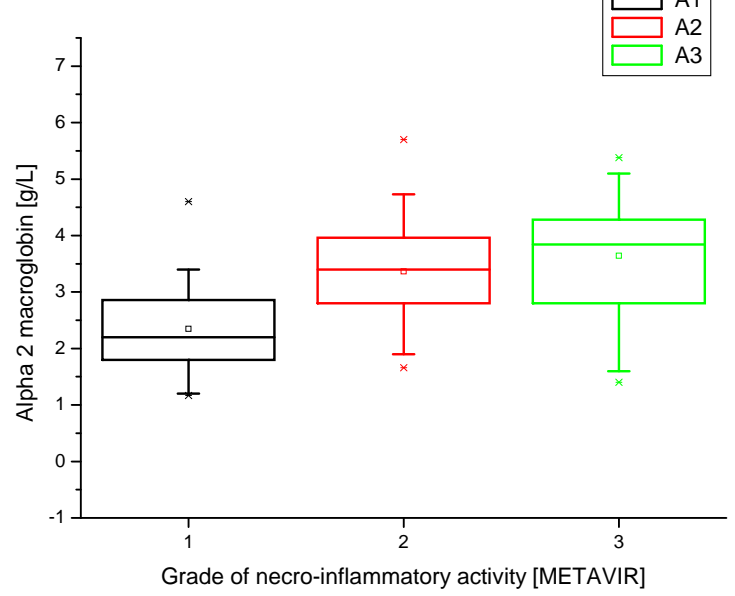

$1 \mathrm{a}$

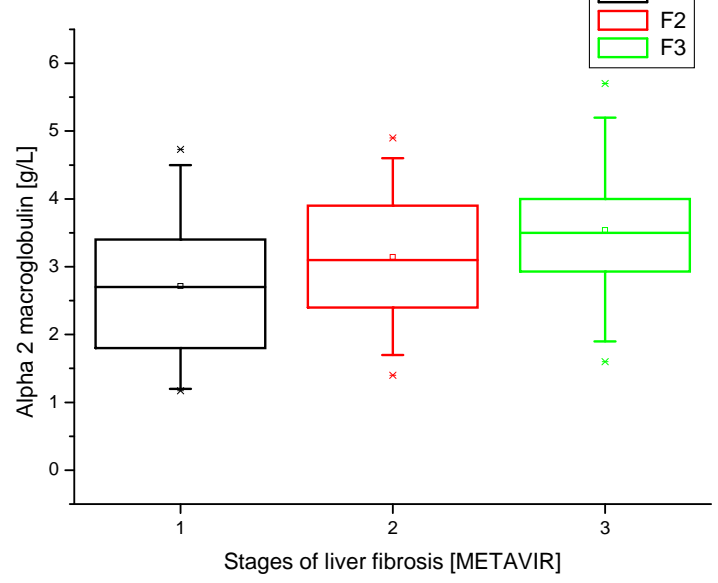

$1 \mathrm{~b}$

Figure 1. A2MG levels according to different parameters: a) grades of hepatic necro-inflammation and b) liver fibrosis stages

\section{A2MG and non-invasive scores for liver fibrosis assessment}

A significant correlation was found between A2MG and APRI, FIB-4, SOS FS and GAMMAGEN, which was low with APPRI, moderate (with Fib-4 and GAMAGEN) and very high with SOS FS (Table 64). 
Table 6. Correlation coefficients ( $r$ ) between A2MG and liver fibrosis scores

\begin{tabular}{|c|c|c|}
\hline Parameters & A2MG & $\mathbf{P}$ \\
\hline$(n=129)$ & 0.433 & 0.000 \\
\hline FIB $4 \quad(n=129)$ & 0.532 & 0.000 \\
\hline SOS FS $(n=129)$ & 0.925 & 0.000 \\
\hline GAMAGEN $(n=98)$ & 0.681 & 0.000 \\
\hline
\end{tabular}

\section{$\underline{A 2 M G}$ and age}

There was a moderate positive correlation between A2MG level and age $(r=0.618 ; p=0.000)$. Mean A2MG value in patients with age $<40$ years; $40-49$ years and $\quad 50$ was: $2.23 \quad 0.72 \mathrm{~g} / \mathrm{L} ; 3.14 \quad 0.79 \mathrm{~g} / \mathrm{L}$; $3.770 .83 \mathrm{~g} / \mathrm{L}$, respectively. The mean A2MG levels in the cescribed 3 age groups were statistically different $(p<0.001)$ - figure 2.

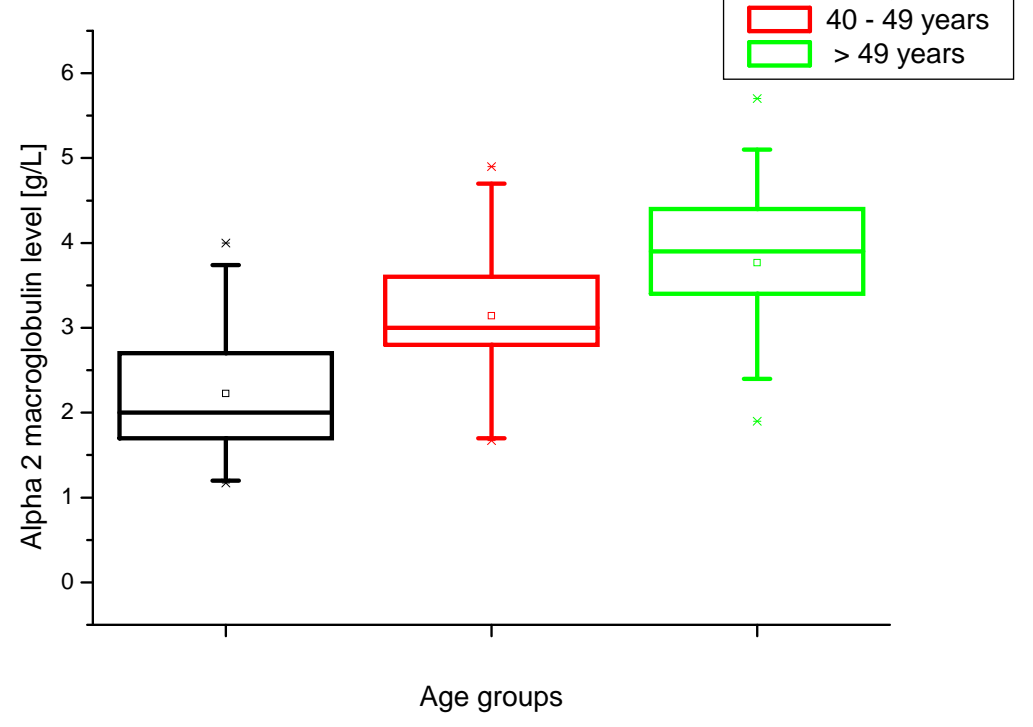

Figure 2. A2MG levels according to age.

\section{Discussion}


Our results demonstrated positive correlations $(r>0.300)$ between A2MG and AST, IgG as well as a negative one between A2MG and haptoglobin. On the other hand A2MG positively correlated with hepatic necro-inflammation and fibrosis. These findings were rather expected as in previous studies the above parameters were identified to be important serum biomarkers of liver fibrosis $(5,6,20)$. For these reasons they have been included in different combinations in several scoring systems for non-invasive assessment of fibrosis. In the present study we did not find a correlation between A2MG and albumin, globulins, CRP, TNF-alpha and pro-hepcidin.

Interestingly, we found a very low correlation between A2MG and platelet count. This was probably due to the relatively low proportion of patients with cirrhosis and advanced fibrosis (F3) in our serial of patients which was only $6 / 129(4.7 \%)$ and $41 / 129(31.8 \%)$, respectively. This fact clearly illustrates the limitations of all scoring systems that are based on platelet count such as APRI and FIB-4. In our study a very low correlation was found between fibrosis stage on liver biopsy and both APRI $(r=0.270)$ and FIB-4 $(r=$ 0.262). On the other hand we found a positive correlation between $A 2 M G$ and fibrosis stage $(r=0.325)$.

Together, all this data clearly suggest that A2MG is the most important serum biomarker for liver fibrosis, which was also confirmed by multivariate logistic regression analysis (5). In this regard scoring systems that include A2MG, such as FibroTest, Hepascore and SOS FS, reflect more accurately liver fibrosis at least in a subset of patients without cirrhosis and advanced fibrosis. However, some of these scoring systems are too complicated or are patented and thus are not widely available. In this situation, it is quite wise to use A2MG for follow-up of CHC patients during antiviral therapy, as it is highly reliable. In our study we found a very high correlation between A2MG and SOS FS $(r=0.925)$.

And finally, a moderate positive correlation between A2MG and patient age is a very important point as fibrosis rapidly progresses in elder patients. Probably, for this reason we observed a higher correlation between A2MG and FIB-4 ( $r=0.532)$ than between A2MG and APRI $(r=0.434)$, as FIB-4 includes patients' age in addition to platelet count and AST. This is also confirmed by the moderate positive correlation between FIB-4 and age $(r=0.518)$.

\section{Conclusion}

In conclusion our study confirms that A2MG is an informative serum biomarker that reflects liver fibrosis and fibrogenesis in chronic hepatitis $C$. It can be used in combination with haptoglobin, IgG and AST in scoring systems for non-invasive assessment of liver fibrosis and figrogenesis. Further studies are needed for the development of simpler, informative and widely available scoring systems for everyday clinical practice.

\section{References}

1. European Association for Study of Liver. EASL Clinical Practice Guidelines: management of hepatitis C virus infection. J Hepatol. 2014; 60 (2): 392-420.

2. Esteban J, Sauleda S, Quer J. The changing epidemiology of hepatitis C virus infection in Europe. J Hepatol 2008; 48: 148-162. 
3. Huang CF, Hsieh MY, Yang JF, et al. Serum hs-CRP was correlated with treatment response to pegylated interferon and ribavirin combination therapy in chronic hepatitis $C$ patients. Hepatol Int. 2010; 4:3: 621-627.

4. Kilic $\mathrm{T}$, Jneid $\mathrm{H}$, Ural $\mathrm{E}$,.et al. Impact of the metabolic syndrome on high-sensitivity $\mathrm{C}$ reactive protein levels in patients with acute coronary syndrome. Atherosclerosis 2009; 207: 591-596.

5. Imbert-Bismut F, Ratziu V, Pieroni L, et al. Biochemical markers of liver fibrosis in patients with hepatitis C virus infection: a prospective study. Lancet. 2001; 7:357:9262) 1069-1075.

6. Ho AS, Cheng CC, Lee SC, et al. Novel biomarkers predict liver fibrosis in hepatitis C patients: alpha 2 macroglobulin, vitamin D binding protein and apolipoprotein Al. J Biomed Sci. 2010; 17: 58 .

7. Tan TC, Crawford DH, Franklin ME, et al. The serum hepcidin: ferritin ratio is a potential biomarker for cirrhosis. Liver Int. 2012; 32:(9: 1391-1399.

8. Wai CT, Greenson JK, Fontana RJ, et al, A simple noninvasive index can predict both significant fibrosis and cirrhosis in patients with chronic hepatitis C. Hepatology. 2003; 38 :(2:: 518-526.

9. Sterling RK, Lissen E, Clumeck N, et al. Development of a simple noninvasive index to predict significant fibrosis in patients with HIV/HCV coinfection. Hepatology. 2006; 43:(6:: 1317-1325.

10. Vallet-Pichard A, Mallet $\mathrm{V}$, Nalpas B, et al. FIB-4: an inexpensive and accurate marker of fibrosis in HCV infection. comparison with liver biopsy and fibrotest. Hepatology. 2007; 46:1:32-36.

11. Oguz A, Atay AE, Tas A, et al. Predictive role of acute phase reactants in the response to therapy in patients with chronic hepatitis C virus infection. Gut Liver. 2013; 7:1:82-88.

12. Naveau S, Poynard T, Benattar C, et al. Alpha-2-macroglobulin and hepatic fibrosis. Diagnostic interest. Dig Dis Sci 1994; 39: 2426-2432.

13. 13. Tiggelman AM, Boers $\mathrm{W}$, Moorman AF, et al. Localization of alpha 2-macroglobulin protein and messenger RNA in rat liver fibrosis: evidence for the synthesis of alpha 2macroglobulin within Schistosoma mansoni egg granulomas. Hepatology 1996; 23: 1260-1267.

14. Kawser CA, Iredale JP, Winwood PJ, Arthur MJ. Rat hepatic stellate cell expression of alpha-2macroglobulin is a feature of cellular activation: implications for matrix remodelling in hepatic fibrosis. Clin Sci 1998; 95: 179-86.

15. Bacq Y, Schillio Y, Brechot JF, et al.. Decrease of haptoglobin serum level in patients with chronic viral hepatitis C. Gastroenterol Clin Biol 1993; 17: 364-369.

16. Louagie HK, Brouwer JT, Delanghe JR, et al. Haptoglobin polymorphism and chronic hepatitis C. J Hepatol 1996; 25: 10-14. 
17. Sakumoto I, Kikuchi N, Takane T, et al.. Studies on the relationship between the histological changes and liver cell function or plasma proteins in hepatic diseases. Gastroenterol Jpn 1976; 11: 224-236.

18. Rossi E, Adams L, Prins A, et al. Validation of the FibroTest biochemical markers score in assessing liver fibrosis in hepatitis $C$ patients. Clin Chem 2003; 49: 450-454.

19. 19. Adams LA, Bulsara M, Rossi E, et al.. Hepascore: an accurate validated predictor of liver fibrosis in chronic hepatitis C infection. Clin Chem. 2005; 51:10: 1867-1873.

20. Kosseva O. Serum markers of liver fibrosis and fibrogenesis. PhD thesis. Sofia 2007.

21. Koenig W, Khuseyinova N, Baumert J, Meisinger C. Prospective study of high-sensitivity Creactive protein as a determinant of mortality: results from the MONICA/KORA Augsburg Cohort Study, 1984-1998. Clin Chem 2008; 54: 335-342.

22. Kushner I. The phenomenon of the acute phase response. Ann NY Acad Sci 1982; 389: 39-48.

Kalabay L, Nemesanszky E, Csepregi A, et al. Paradoxical alteration of acute-phase protein levels in patients with chronic hepatitis C treated with IFN-alpha2b. Int Immunol 2004; 16: 51-54.

23. Tsui JI, Whooley MA, Monto A, et al. Association of hepatitis C virus seropositivity with inflammatory markers and heart failure in persons with coronary heart disease: data from the Heart and Soul study. J Card Fail 2009; 15: 451-456.

24. Reingold J, Wanke C, Kotler D, et al. Association of HIV infection and HIV/HCV coinfection with C reactive protein levels: the fat redistribution and metabolic change in HIV infection (FRAM) study. J Acquir Immune Defic Syndr 2008; 48: 142-148.

25. Floris-Moore M, Howard AA, Lo $Y$, et al. Hepatitis $C$ infection is associated with lower lipids and high-sensitivity C-reactive protein in HIV-infected men. AIDS Patient Care STDS 2007; 21 : 479 491.

26. Yelken B, Gorgulu N, Caliskan Y, et al. Association between chronic hepatitis C infection and coronary flow reserve in dialysis patients with failed renal allografts. Transplant Proc 2009; 41: 1519-1523.

27. Nascimento MM, Bruchfeld A, Suliman ME, et al. Effect of hepatitis C serology on C-reactive protein in a cohort of Brazilian hemodialysis patients. Braz J Med Biol Res 2005; 38: 783-788.

28. Larrea E, Garcia N, Qian C, et al. Tumor necrosis factor alpha gene expression and the response to interferon in chronic hepatitis C. Hepatology. 1996; 23 :2) 210-217.

29. Bedossa P, Poynard T. An algorithm for the grading of activity in chronic hepatitis $\mathrm{C}$. The METAVIR Cooperative Study Group. Hepatology 1996; 24: 289-293.

We declare that we have no conflict of interest. 
This material is consistent with the Ethical Guidelines for Good Research Practice.

\section{Corresponding author}

Evelina Atanasova

University Hospital "St. Ivan Rilski", Clinic of Gastroenterology

1431 Sofia, Bulgaria,

15 "Acad. Ivan Geshov" blvd.

e-mail: eva_gatanasova@abv.bg

Fax number: +35928510615 